\title{
Solving Transportation Problems Using The Best CANDIDATES METHOD
}

\author{
Abdallah A. Hlayel, Mohammad A. Alia \\ Department of Computer Information Systems, \\ Faculty of Science and Information Technology, \\ Al-Zaytoonah University of Jordan, P.O. Box 130 Amman 11733 Jordan \\ hlayel@zuj.edu.jo, dr.m.alia@zuj.edu.jo
}

\begin{abstract}
Problem statement: The optimization processes in mathematics, computer science and economics are solving effectively by choosing the best element from set of available alternatives elements. The most important and successful applications in the optimaization refers to transportation problem (TP), that is a special class of the linear programming $(L P)$ in the operation research (OR). Approach: The main objective of transportation problem solution methods is to minimize the cost or the time of transportation. Most of the currently used methods for solving transportation problems are trying to reach the optimal solution, whereby, most of these methods are considerd complex and very expansive in term of the execution time. In this study we use the best candidate method (BCM), in which the key idea is to minimize the combinations of the solution by choosing the best candidates to reach the optimal solution. Results: Comparatively, applying the BCM in the proposed method obtains the best initial feasible solution to a transportation problem and performs faster than the existing methods with a minimal computation time and less complexity. The proposed methods is therefore an attractive alternative to traditional problem solution methods. Conclusion/Recommendations: The BCM can be used successfully to solve different business problems of distrbution products that is commonly referd to a transportation problems.
\end{abstract}

\section{KEYWORDS}

Operation research, transportation problem, linear programming, optimization problems, transportation model, Vogel's approximation method, best candidates method, optimal solution, solving optimization, and objective function.

\section{INTRODUCTION}

Ttransportation problem is concerned with the optimal pattern of the product units' distribution from several original points to several destinations. Suppose there are $m$ points of original $A_{1}, \ldots$ , $A_{i}, \ldots, A_{m}$ and $n$ destinations $B_{1}, \ldots, B_{j}, \ldots, B_{n}$. The point $A_{i}(i=1, \ldots, m)$ can supply $a_{i}$ units, and the destination $B_{j}(j=1, \ldots, n)$ requires $b_{j}$ units (see Equation 1).

$\sum_{i=1}^{m} a_{i}=\sum_{j=1}^{n} b_{i}$

Whereby, the cost of shipping a unit from $A_{j}$ to $B_{j}$, is computed as $c_{i j}$. As well as, the problem in determining the optimal distribution pattern consists the pattern for which shipping costs are at a minimum. Moreover, the requirements of the destinations $B_{j}, j=1, \ldots, n$, must be satisfied by the supply of available units at the points of origin $A_{j}, i=1, \ldots, m$. 
As shown by Equation 2, if $x_{i j}$ is the number of units that are shipped from $A_{i}$ to $B_{j}$, then the problem in determining the values of the variables $x_{i j}, i=1, \ldots, m$ and $j=1, \ldots, n$, should minimize the total of the shipping costs.

$\sum_{i=1}^{m} \sum_{j=1}^{n} c_{i j} x_{i j}$

While,

$$
\begin{aligned}
& \sum_{j=1}^{n} x_{i j}=a_{i} ; i=1, \ldots, m \\
& \sum_{i=1}^{m} x_{i j}=b_{j} ; j=1, \ldots, n \\
& x_{i j}>0 ; i-1, \ldots, m ; \text { and } j-1, \ldots, n
\end{aligned}
$$

Mathematically, the transportation problem can be represented as a linear programming model. Since the objective function in this problem is to minimize the total transportation cost as given by Equation 3.

$Z=c_{1:} x_{11}+c_{12} x_{12}+\cdots+c_{m n} x_{m n}$

Equation 3 is a mathematical formulation of a transportation problem that can adopt the linear programming (LP) technique with equality constraints. LP technique can be used in different product areas such as oil plum industry [1]. However, The LP technique can be generally used by genetic algorithm such as Sudha at el. article [2]. The transportation solution problem can be found with a good success in the improving the service quality of the public transport systems [3]. Also it is found in Zuhaimy Ismail at el. article [4]. As well as, the transportation solution problem is used in the electronic commerce where the area of globalization the degree of competition in the market article [5], and it can be used in a scientific fields such as the simulated data for biochemical and chemical Oxygen demands transport [6], and many other fields.

However, there are several different algorithms to solve transportation problem that represented as LP model. Among these are the known algebraic procedures of the simplex method, which may not be the best method to solve the problem. Therefore, more efficient and simpler procedures have been improved to solve transportation problems. Typically, the standard scenario for solving transportation problems is working by sending units of a product across a network of highways that connect a given set of cities. Each city is considered as a source $(\mathrm{S})$ in that units will be shipped out from, while units are demanded there when the city is considered as a sink (D). In this scenario, each sink has a given demand, the source has a given supply, and the highway that connects source with sink as a pair has a given transportation cost/(shipment unit). Figure 1 visualizes the standard scenario for cities on the highway in the form of a network.

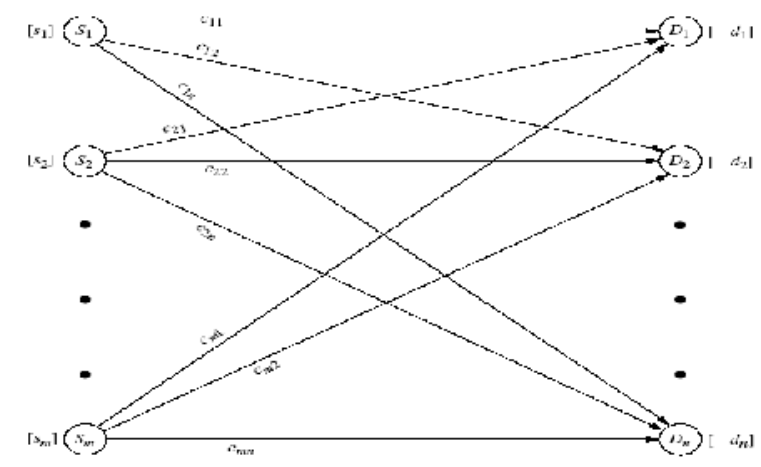

Figure1. Network flow model of the transportation problem. 
As shown by Figure 1, the problem is to determine an optimal transportation scheme that is to minimize the total of the shipments cost between the nodes in the network model, subject to supply and demand constraints. As well as, this structure arises in many applications such as; the sources represent warehouses and the sinks represent retail outlets.

Moreover, Ad-hoc networks are designed dynamically by group of mobile devices. In Ad-hoc network, nodes between source and destination act as a routers so that source node can communicate with the destination node [7].

\section{MATERIALS AND METHOD}

Transportation model: In a transportation problem, we are focusing on the original points. These points may represent factories to produce items, and to supply a required quantity of the products to a certain number of destinations. This process must be done successfully in such a way as to maximize the profit or minimize the cost transportation. Therefore, the places of production and supply are collected as the original points and the destinations respectively. Sometimes the original and destinations points are also termed as sources and sinks. However, to illustrate a typical transportation model, suppose that $m$ factories supply certain items to $n$ warehouses. As well as, let factory $i(i=1,2, \ldots, m)$ produces $a_{\mathrm{i}}$ units, and the warehouse $j(j=1$, $2, \ldots, n)$ requires $b_{\mathrm{j}}$ units. Furthermore, suppose the cost of transportation from factory $i$ to warehouse $j$ is $c_{\mathrm{ij}}$. The decision variables $x_{\mathrm{ij}}$ is being the transported amount from the factory $i$ to the warehouse $j$. Typically, our objective is to find the transportation pattern that will minimize the total of the transportation cost (see Table 1).

Table 1: The model of a transportation problem

\begin{tabular}{|l|l|l|l|l|}
\hline $\begin{array}{l}\text { Origins } \\
\text { (Factories) }\end{array}$ & $\begin{array}{l}\text { Destinations } \\
\text { (Warehouses) }\end{array}$ & Available \\
\hline & $12 \ldots \ldots \ldots \mathrm{n}$ & & \\
\hline 1 & $c_{11}$ & $c_{12}$ & $c_{1 n}$ & $a_{1}$ \\
\hline 2 & $c_{21}$ & $c_{22}$ & $c_{2 n}$ & $a_{2}$ \\
\hline$\ldots$ & $\ldots$ & $\ldots$ & $\ldots$ & $\ldots$ \\
\hline $\mathrm{m}$ & $c_{m 1}$ & $c_{m 2}$ & $c_{m n}$ & $a_{m}$ \\
\hline Required & $b_{1}$ & $b_{2}$ & $b_{n}$ & \\
\hline
\end{tabular}

Algorithms for solving: ther are several algorithm for solving ttransportation problems which are based on different of special linear programming methods, among these are:

1. Northwest Corner method

2. Minimum cost method

1. Genetic algorithm

3. Vogel's approximation method

4. Row Minimum Method

5. Column Minimum Method

Basically, these methods are different in term of the quality for the produced basic starting solution and the best starting solution that yields smaller objective value. In this study, we used the Vogel's approximation method, since it generally produces better starting solutions than other solving methods; as well we have used the BCM solution steps [8]. 
Computer Science \& Engineering: An International Journal (CSEIJ), Vol.2, No.5, October 2012

\section{Vogel's Approximation Method (VAM):}

Generally, VAM can be summarized by the following three main steps [9]:

i. The result of subtracting the smallest unit cost element in the row/column (cell) from the immediate next smallest unit cost element in the same row/column is determining a penalty measure for the target row/column.

ii. This step includes the following sub-steps:

a. Identify the row or the column that includes the largest penalty.

b. Break ties arbitrarily.

c. As much as possible, the lowest cost row/column (cell) in the row or column should be allocated with the highest difference.

d. Adjust the supply and demand, and then cross out the satisfied row or column.

e. If a row and column are satisfied simultaneously, then only one of them is crossed out, as well the remains rows or columns are assigned to supply as zero (demand).

iii. Finally, the result should be computed as follows:

a. If a row or a column is assigned as zero supply, or demand remains uncrossed out, then stop the process.

b. If one row/column with positive supply (demand) remains uncrossed out, then determine the basic variables in the row/column by the lowest cost method, and then stop.

c. If all the uncrossed out rows and columns have (remaining) zero supply and demand then determine the zero basic variables by the lowest cost method and stop.

d. Otherwise, go to step (i).

\section{The Best Candidates Method (BCM):}

BCM process includes three steps, these steps are shown as follows [8]:

Step1: Prepare the BCM matrix, If the matrix unbalanced, then the matrix will be balanced without using the added row or column candidates in solution procedure.

Step2: Select the best candidates, that is for minimizing problems to the minimum cost, and maximizing profit to the maxumum cost. Therefore, this step can be done by electing the best two candidates in each row. If the candidate repeated more than two times, then the candidate should be elected again. As well as ,the columns must be checked such that if it is not have candidates so that the candidates will be elected for them. However, if the candidate is repeated more than one time, the elect it again.

Step3: Find the combinations by determining one candidate for each row and column, this shoud be done by starting from the row that have the least candidates, and then delete that row and column. If there is situation that have no candidate for some rows or columns, then directly elect the best available candidate. Repeat Step 3 by determining the next candidate in the row that started from. Compute and compare the summation of candidates for each combination. This is to determine the best combination that give the optimal solution.

\section{PROPOSED METHOD}

In this study, we proposed a new solving method for transportation problems by using BCM. The proposed method must operate the as following:

Step1: We must check the matrix balance, If the total supply is equal to the total demand, then the matrix is balanced and also apply Step 2. If the total supply is not equal to the total demand, then we add a dummy row or column as needed to make supply is equal to the demand. So the transportation costs in this row or column will be assigned to zero. 
Computer Science \& Engineering: An International Journal (CSEIJ), Vol.2, No.5, October 2012

Step2: Appling BCM to determine the best combination that is to produce the lowest total weight of the costs, where is one candidate for each row and column.

Step3: Identify the row with the smallest cost candidate from the choosen combination. Then allocate the demand and the supply as much as possible to the variable with the least unit cost in the selected row or column. Also, we should adjust the supply and demand by crossing out the row/column to be then assigned to zero. If the row or column is not assigned to zero, then we check the selected row if it has an elemnt with lowest cost comparing to the determined element in the chossen combination, then we elect it.

Step4:Elect the next least cost from the choosen combination and repeat Step 3 untill all columns and rows is exhusted.

Problem: Finding the optimal cost for the problem of assigning five jobs to five persons.

\section{Solution:}

Step1: in this problem, the matrix is unbalanced, where the total of supply is not equal to the total of demand (Table 2). Here we add a dummy row to make supply is equal to the demand, so the transportation costs in this row will be assigned to zero (Table 3).

Table 2:the proposed method -process 1

\begin{tabular}{|l|l|l|l|l|l|l|}
\hline Plant & D1 & D2 & D3 & D4 & Supply \\
\hline S1 & 10 & 30 & 25 & 15 & 14 & \\
\hline S2 & 20 & 15 & 20 & 10 & 10 & \\
\hline S3 & 10 & 30 & 20 & 20 & 15 & \\
\hline S4 & 30 & 40 & 35 & 45 & 12 & \\
\hline Demand & 10 & 15 & 12 & 15 & $\mathbf{5 2}$ & $\mathbf{5 1}$ \\
\hline
\end{tabular}

Table 3: the proposed method -process 2

\begin{tabular}{|l|l|l|l|l|l|l|}
\hline Plant & D1 & D2 & D3 & D4 & \multicolumn{2}{|l|}{ Supply } \\
\hline S1 & 10 & 30 & 25 & 15 & 14 & \\
\hline S2 & 20 & 15 & 20 & 10 & 10 & \\
\hline S3 & 10 & 30 & 20 & 20 & 15 & \\
\hline S4 & 30 & 40 & 35 & 45 & 12 & \\
\hline S5 & 0 & 0 & 0 & 0 & 1 & \\
\hline Demand & 10 & 15 & 12 & 15 & 52 & 52 \\
\hline
\end{tabular}

Step2: By using BCM, we determine the best combination that will produce the lowest total weight of the costs, where is one candidate for each row and column. The result from appling BCM shown in (Table 4).

Table 4: the proposed method -process 3

\begin{tabular}{|l|l|l|l|l|l|l|}
\hline Plant & D1 & D2 & D3 & D4 & \multicolumn{2}{|l|}{ Supply } \\
\hline S1 & 10 & 30 & 25 & 15 & 14 & \\
\hline S2 & 20 & 15 & 20 & 10 & 10 & \\
\hline S3 & 10 & 30 & 20 & 20 & 15 & \\
\hline S4 & 30 & 40 & 35 & 45 & 12 & \\
\hline S5 & 0 & 0 & 0 & 0 & 1 & \\
\hline Demand & 10 & 15 & 12 & 15 & $\mathbf{5 2}$ & $\mathbf{5 2}$ \\
\hline
\end{tabular}


Computer Science \& Engineering: An International Journal (CSEIJ), Vol.2, No.5, October 2012

Step3: We identify the row with the smallest cost candidate from the choosen combination. Then we allocate the supply and the demand to the variable with the least unit cost in the selected row and column. Also, we should adjust the supply and the demand by crossing out the row/column to be then assigned to zero (Table 5). If the column is assigned to zero, then we elect the cost in the cell $(\mathrm{d} 3, \mathrm{~s} 3)$ because it has a lower cost than the determined cell $(\mathrm{d} 3, \mathrm{~s} 4)$ from the choosen combination (Table 6) .

Table 5: the proposed method -process 4

\begin{tabular}{|c|c|c|c|c|c|}
\hline Plant & D1 & D2 & D3 & D4 & Supply \\
\hline S1 & 10 & 30 & 25 & 15 & 14 \\
\hline S2 & 20 & (15) & 20 & 10 & 10 \\
\hline S3 & (10) 10 & 30 & 20 & 20 & 155 \\
\hline S4 & 30 & 40 & (35) & 45 & 12 \\
\hline S5 & 0 & 0 & 0 & 0 & 1 \\
\hline Demand & 10 & 15 & 12 & 15 & $52 \quad 52$ \\
\hline
\end{tabular}

Table 6: the proposed method -process 5

\begin{tabular}{|c|c|c|c|c|c|}
\hline Plant & D1 & D2 & D3 & D4 & Supply \\
\hline S1 & 10 & 30 & 25 & 15 & 14 \\
\hline $\mathrm{S} 2$ & 20 & 15) & 20 & 10 & 10 \\
\hline S3 & (10) 10 & 30 & 205 & 20 & 15.50 \\
\hline S4 & 30 & 40 & 35 & 45 & 12 \\
\hline S5 & 0 & 0 & 0 & 0 & 1 \\
\hline Demand & 10 & 15 & 12 & 15 & $52 \quad 52$ \\
\hline
\end{tabular}

Step4: We elect the next least cost from the choosen combination and repeat Step 3 untill all columns and rows are exhusted (Table 7).

Table 7: the proposed method -process 6

\begin{tabular}{|c|c|c|c|c|c|}
\hline Plant & D1 & D2 & D3 & D4 & Supply \\
\hline S1 & 10 & 30 & 25 & (15) 14 & 140 \\
\hline S2 & 20 & (15) 10 & 20 & 10 & 100 \\
\hline S3 & (10) 10 & 30 & 205 & 20 & $15 \% 0$ \\
\hline S4 & 30 & 405 & (35) 7 & 45 & 120 \\
\hline S5 & 0 & 0 & 0 & $\begin{array}{ll}0 & 1\end{array}$ & 10 \\
\hline Demand & 10 & 15 & 12 & 15 & $52 \quad 52$ \\
\hline
\end{tabular}

We found that the result by using $\mathrm{BCM}$ as:

$15 * 14+15 * 10+10 * 10+20 * 5+40 * 5+35 * 7=1005$

\section{RESULTS AND DISCUSSION}

Actually, using BCM based on the best candidates election is to minimize the number of combinations solutions. As we can obtain the combinations without any intersect means, such that; one candidate for each row and column. Eficentaly, BCM works to obain the optimal 
Computer Science \& Engineering: An International Journal (CSEIJ), Vol.2, No.5, October 2012

solution or the closest to optimal solution than other available methods. Since it has a better starting solution.

\section{CONCLUSION}

In this study, we have proposed a BCM for solving transportation problems, because of its wide applicability in different area. Whereby, it refers to choose the best distrubtion of cost or time. The BCM obtained the optimal solution or the closest to optimal solution with a minimum computation time. As well as, using BCM will reduces the complexity with a simple and a clear solution manner which is can be easily used on different area for optimization problems.

\section{ACKNOWLEDGEMENTS}

The authors would like to thank Al Zaytoonah University of Jordan for supporting this study.

\section{REFERENCES}

[1] Elaine L.Y. Man and Adam Baharum , 2011. A Qualitative Approach of Identifying Major Cost Influencing Factors in Palm Oil Mills and the Relations towards Production Cost of Crude Palm Oil. American Journal of Applied Sciences, 8: 441-446. DOI: 10.3844/ajassp.2011.441.446.

[2] Sudha, S.V. and K. Thanushkodi, 2012. A genetic based neuro fuzzy technique for process grain sized scheduling of parallel jobs. J. Comput. Sci., 8:48-54. DOI: 10.3844/jcssp.2012.48.54

[3] Amir, S., H.Z. Aashtiani and K.A. Mohammadian, 2009. A Shor-term Management strategy for Improving transit network efficiency. Am. J. Applied Sci., 6: 241-246. DOI: 10.3844/ajassp.2009.241.246.

[4] Zuhaimy Ismail, Diyana A. Mahad and Tan S. Ching, 2011. Modeling of Multi-Level Capacitated Lot-Size Scheduling Problem. American Journal of Applied Sciences, 8: 290-296. DOI: 10.3844/ajassp.2011.290.296.

[5] A. Norozi , M.K.A. Ariffin and N. Ismail , 2010. Application of Intelligence Based Genetic Algorithm for Job Sequencing Problem on Parallel Mixed-Model Assembly Line. American Journal of Engineering and Applied Sciences, 3: 15-24. DOI: 10.3844/ajeassp.2010.15.24.

[6] Eisakhani, M., M.P. Abdullah, O.A. Karim and A. Malakahmad, 2012. Validation of MIKE 11 model simulated, data for biochemical and chemical oxygen demands transport. Am. J. Applied Sci., 9: 382 387. DOI: 10.3844/ajassp.2012.382.387.

[7] Suganthi, P. and A. Tamilarasi, 2012. Impact of malicious nodes under different route refresh intervals in ad hoc network. Am. J. Applied Sci., 9:18-23. DOI: 10.3844/ajassp.2012.18.23

[8] Ahmad, H.A., 2012. The best candidates method for solving optimization problems. J. Comput. Sci., 8: 711-715. DOI: 10.3844/jcssp.2012.711.715.

[9] Taghrid, I., Mohamed G., Iman S., 2009. Solving Transportation Problem Using Object-Oriented Model. IJCSNS International Journal of Computer Science and Network Security, VOL.9 No.2. 


\section{AUTHORS}

Dr. Abdallah Hlayel is an Assistance professor at the computer information systems department, Faculty of Science and Information Technology, Al Zaytoonah University of Jordan. He received the B.Sc. \& M.Sc. in Engineering Science/Computer from Faculty of Automatics and Computer Engineering, Kiev Institute of Civil Aviation Engineers, in 1990. He obtained his Ph.D. in Engineering Science/Computer from National Academy of Science of Ukraine, in 1994. During 1995 until 1997, he worked at Philadelphia University as a lecturer in the Department of Computer Engineering $\backslash$ Electrical. Then, he worked as a lecturer at Al Zaytoonah University of Jordan from 1997 until now. From 2000 to 2003 he was the

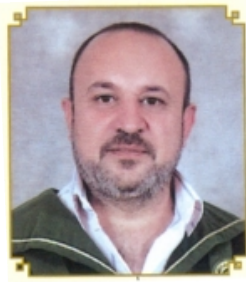
Head of Computer Centre - Al Zaytoonah University. His research interests are in the field of operation research, dynamic programming, computer systems, antennas and their sets, administration systems and elements of computer.

Dr. Mohammad Alia is an Assistance professor at the computer information systems department, Faculty of Science Computer and information technology, Al Zaytoonah University of Jordan. He received the B.Sc. degree in Science from the Alzaytoonah University, Jordan, in 2000. He obtained his Ph.D. degree in Computer Science from University Science of Malaysia, in 2008. During 2000 until 2004, he worked at AlZaytoonah University of Jordan as an instructor of Computer sciences and Information Technology. Then, he worked as a lecturer at Al-Quds University in Saudi Arabia from 2004 - 2005. Currently he is working as a Chair of Computer Information

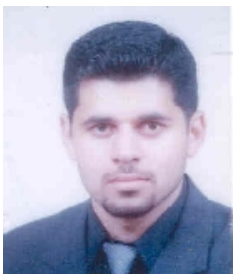
Systems dept. at Al Zaytoonah University of Jordan. His research interests are in the field of Cryptography, and Network security. 